\title{
Results of the research into the harmonics of loads connected to the nodes of high voltage network
}

\author{
L. I. Kovernikova \\ The Siberia Branch of the Russian Academy of Sciences \\ Energy Systems Institute \\ 130, Lermontov Str., Irkutsk, 664033, (Russia) \\ Phone: +7 3952500646 ext. tel. 232, fax: +7 3952 426796, e-mail: kovernikova @isem.sei.irk.ru
}

\begin{abstract}
The paper presents the results of the studies on measured parameters of harmonics for the complex loads connected to the nodes of the high voltage network. The measurements were made at four nodes of the $110-220 \mathrm{kV}$ network. The complex loads include residential, commercial, industrial and tractional consumers. The considered nodes are the connection points of the railway substations to the feeding network. The paper demonstrates the specific features of the harmonic conditions.
\end{abstract}

\section{Key words}

Power quality, harmonics, high voltage network, measurement, statistical analysis

\section{Introduction}

Experimental studies on the parameters of harmonics in electrical networks of different voltages have been conducted since long ago. The results of the studies for the low- and medium-voltage networks are presented in comprehensive and well known publications [1-5]. The publications present the results of research on harmonics in the networks with different loads. In the last decade researchers have made a lot of measurements of harmonic parameters which can be explained by the appearance and wide use of new nonlinear electric equipment and increase in the harmonic currents and voltages in the network. For example, the authors of [6] present the results of measurements at the network node which feeds the nonlinear loads of affiliated stations. The authors of [7] show the measurements of harmonics generated by energy saving lamps. The paper [8] presents the level of harmonics measured in the medical center and their negative impact on the operation of medical equipment. In [9] the authors show the results of large-scale measurements of power quality indices in the $110 \mathrm{kV}$ networks of Siberia. The measurements were carried out in the electrical networks covering 23 percent of Russia's territory. The measurements confirmed the presence of harmonics in the electrical networks. In many cases the level of harmonic voltage exceeded the limits established in [10].

This paper presents the results of measurements and analysis of harmonic parameters for complex loads connected to the nodes of high voltage networks. The complex loads include residential, commercial, industrial and tractional consumers. The load has a large share of nonlinear equipment. The goal of the research is to study the properties of harmonics for the development of models of loads at the nodes of high voltage network, which will be used for the calculation of nonsinusoidal network conditions.

\section{Object of Research}

The measurements were made at four nodes of the network, which represent the high voltage buses of traction substations: $220 \mathrm{kV}$ Mysovaya (M), $110 \mathrm{kV}$ Tataurovo (T), $220 \mathrm{kV}$ Zaigraevo (Z), and $220 \mathrm{kV}$ Novoilinsky (N). Substation T is situated $126 \mathrm{~km}$ from substation $\mathrm{M}$, substation $\mathrm{Z}-86 \mathrm{~km}$ from substation $\mathrm{T}$, and substation $\mathrm{N}$ is situated $47 \mathrm{~km}$ from substation $\mathrm{Z}$. Each node is connected to a 40 MVA three-phase threewinding transformer and transmission lines running to the neighboring traction substations and other nodes in the high voltage network. The medium voltage winding of the transformer $(25 \mathrm{kV})$ feeds the traction load. The low voltage winding $(6$ or $11 \mathrm{kV})$ supplies power to residential, commercial, industrial loads, etc. The scheme of the node is presented in Fig. 1.

In general, the neighboring railway substations are located at a 40-60 km distance from one another. Each section of railway between two substations receives 
power from two sides. The substations appear to be quite evenly distributed along the feeding network. DC engines drive electric locomotives. The engines are powered through single-phase two-pulse rectifier circuits. The rectifier circuits in the $25 \mathrm{kV}$ network cause harmonic currents. The harmonic currents penetrate through transformers into the $110-220 \mathrm{kV}$ feeding network and cause a distortion of the voltage waveform. Thus, the traction load for the high voltage network is nonlinear, unbalanced and distributed.

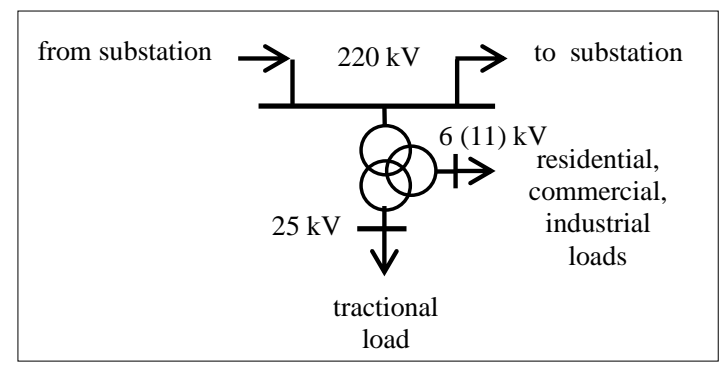

Fig. 1. The scheme of the node of high voltage network

The measurements were carried on the high voltage side of the transformers. Measurements were performed with the aid of the device "Omsk", which measures not only the indices of power quality but also currents, powers and other parameters. Each measurement was performed during 24 hours. The parameters were measured mainly in an interval of 1 minute. For voltage measurements the device was connected through voltage transformers to the high voltage buses. For current measurements the device was connected to current transformers installed at the inputs of high voltage transformers.

\section{Analysis of Measured Parameters}

\section{A. Harmonic Voltages in Terms of the Standard Requirements}

The values of measured phase harmonic voltages at nodes with the probability of 95 percent are presented in Table I and Table II. Letters $\mathrm{a}, \mathrm{b}$, and $\mathrm{c}$ stand for phases. The values exceeding the limits [10] are highlighted in bold. The standards for the values of parameters [10] that are established in Russia have been harmonized with European standards [11].

Table I presents the values $U n$ measured in the conditions when loads are connected to the windings of the mediumand low- voltage transformer. In Table II the values $U n$ correspond to the conditions when load is connected to the low-voltage winding only. The Tables I, II show that the harmonic conditions are asymmetrical and the limits of $U n$ are exceeded at harmonics 3, 5, 7, 9, 23. Comparison of the data from both tables allows the conclusion that the disconnection of traction load at nodes $\mathrm{M}, \mathrm{N}$, and $\mathrm{Z}$ has little influence on the values $U n$. At node $T$ the disconnection of traction load causes a noticeable reduction in the voltages of the $3,5,7$ th harmonics although their values exceed the established limits. It is possible to suppose that the harmonic voltages at each of the nodes result from the impact of multiple nonlinear loads that are powered from one and the same highvoltage network.

Table I. - Measured Values of Un (\%)

\begin{tabular}{|c|c|c|c|c|c|c|c|c|}
\hline \multirow{2}{*}{$\begin{array}{c}\text { No- } \\
\text { de }\end{array}$} & \multicolumn{7}{|c|}{ Harmonic } \\
\cline { 2 - 9 } M-a & 1.2 & $\mathbf{2 . 0}$ & 0.5 & 0.2 & 0.6 & 0.4 & 0.3 & 0.3 \\
\hline M-b & 1.3 & $\mathbf{2 . 1}$ & 0.6 & 0.2 & 0.6 & 0.3 & 0.4 & 0.2 \\
\hline M-c & $\mathbf{1 . 6}$ & $\mathbf{2 . 2}$ & 0.5 & 0.2 & 0.7 & 0.3 & 0.4 & 0.2 \\
\hline N-a & 1.5 & $\mathbf{1 . 7}$ & 0.5 & $\mathbf{0 . 6}$ & 0.7 & 0.3 & 0.4 & 0.2 \\
\hline N-b & 1.3 & $\mathbf{1 . 8}$ & 0.6 & $\mathbf{0 . 6}$ & 0.8 & 0.2 & $\mathbf{0 . 5}$ & 0.2 \\
\hline N-c & $\mathbf{1 . 6}$ & $\mathbf{1 . 7}$ & 0.5 & $\mathbf{0 . 5}$ & 0.6 & 0.3 & 0.4 & 0.3 \\
\hline T-a & $\mathbf{2 . 8}$ & $\mathbf{2 . 6}$ & $\mathbf{1 . 1}$ & $\mathbf{0 . 7}$ & 0.9 & 0.3 & 0.3 & 0.2 \\
\hline T-b & $\mathbf{2 . 5}$ & $\mathbf{3 . 6}$ & 0.8 & $\mathbf{0 . 7}$ & 0.7 & 0.3 & 0.3 & 0.1 \\
\hline T-c & $\mathbf{2 . 8}$ & $\mathbf{3 . 2}$ & 0.7 & $\mathbf{0 . 5}$ & 0.8 & 0.3 & 0.3 & 0.1 \\
\hline Z-a & 1.1 & 1.3 & 0.7 & $\mathbf{0 . 5}$ & 0.5 & 0.4 & 0.3 & 0.2 \\
\hline Z-b & 1.1 & 1.2 & 0.7 & $\mathbf{0 . 6}$ & 0.6 & 0.4 & 0.1 & 0.1 \\
\hline Z-c & $\mathbf{1 . 7}$ & 1.0 & 0.6 & $\mathbf{0 . 5}$ & 0.4 & 0.3 & 0.3 & 0.2 \\
\hline limit & 1.5 & 1.5 & 1 & 0.4 & 1 & 0.7 & 0.4 & 0.4 \\
\hline
\end{tabular}

Table II. - Measured Values of Un (\%)

\begin{tabular}{|c|c|c|c|c|c|c|c|c|}
\hline $\begin{array}{c}\text { No- } \\
\text { de }\end{array}$ & 3 & 5 & 7 & 9 & 11 & 13 & 23 & 25 \\
\hline M-a & 1.2 & $\mathbf{1 . 9}$ & 0.5 & 0.2 & 0.6 & 0.4 & 0.3 & 0.3 \\
\hline M-b & 1.3 & $\mathbf{2 . 1}$ & 0.5 & 0.2 & 0.7 & 0.3 & 0.4 & 0.2 \\
\hline M-c & 1.4 & $\mathbf{1 . 8}$ & 0.4 & 0.2 & 0.8 & 0.3 & 0.3 & 0.2 \\
\hline N-a & 1.3 & $\mathbf{1 . 8}$ & 0.5 & $\mathbf{0 . 6}$ & 0.7 & 0.3 & $\mathbf{0 . 5}$ & 0.2 \\
\hline N-b & 0.9 & $\mathbf{2 . 0}$ & 0.6 & $\mathbf{0 . 6}$ & 0.7 & 0.3 & $\mathbf{0 . 5}$ & 0.3 \\
\hline N-c & $\mathbf{1 . 8}$ & $\mathbf{2 . 0}$ & 0.5 & $\mathbf{0 . 6}$ & 0.5 & 0.3 & $\mathbf{0 . 5}$ & 0.3 \\
\hline T-a & $\mathbf{2 . 2}$ & 1.4 & 0.7 & $\mathbf{0 . 5}$ & 0.8 & 0.5 & 0.3 & 0.1 \\
\hline T-b & $\mathbf{1 . 9}$ & $\mathbf{1 . 6}$ & 0.6 & 0.4 & 0.7 & 0.3 & 0.4 & 0.1 \\
\hline T-c & $\mathbf{2 . 1}$ & 1.2 & 0.6 & $\mathbf{0 . 5}$ & 0.7 & 0.4 & 0.3 & 0.1 \\
\hline Z-a & 1.0 & 1.4 & 0.6 & $\mathbf{0 . 5}$ & 0.5 & 0.4 & 0.1 & 0.1 \\
\hline Z-b & 0.9 & 1.3 & 0.7 & $\mathbf{0 . 6}$ & 0.6 & 0.4 & 0.2 & 0.1 \\
\hline Z-c & $\mathbf{1 . 8}$ & 1.4 & 0.6 & $\mathbf{0 . 5}$ & 0.5 & 0.3 & 0.3 & 0.1 \\
\hline limit & 1.5 & 1.5 & 1 & 0.4 & 1 & 0.7 & 0.4 & 0.4 \\
\hline
\end{tabular}

\section{B. Active and Reactive Powers of the Fundamental Frequency}

The active and reactive powers measured for the complex loads of one phase at node $\mathrm{Z}$ are presented in Fig. 2. They demonstrate a typical character of change in the powers at railway substations. The powers in Fig. 2a represent the total active and reactive powers consumed by the loads that are connected to the medium- and lowvoltage windings of transformers. The curves have an abruptly variable character.

The powers of loads that are connected to the mediumvoltage winding are the total powers of electric locomotives that are powered by the traction network. Figure $2 \mathrm{~b}$ demonstrates the active and reactive power of the loads connected to the low-voltage winding. The values of powers in the graphs change slowly. The powers of loads of the medium-voltage winding exceed the powers of loads of low-voltage winding approximately by 2-4 times. The loads at all the studied nodes have similar power graphs. 


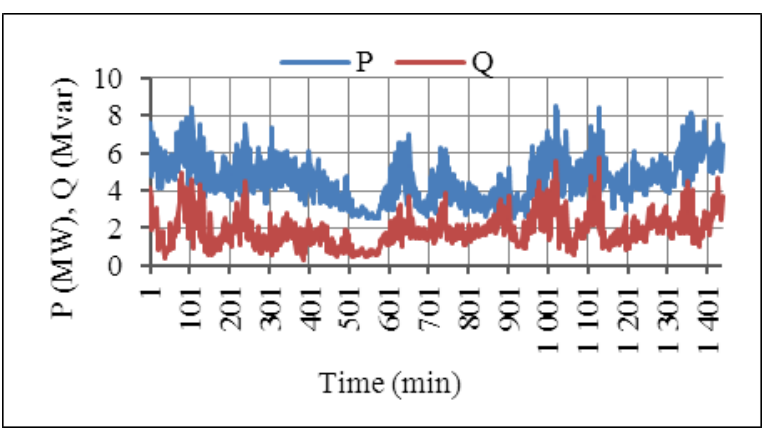

a)

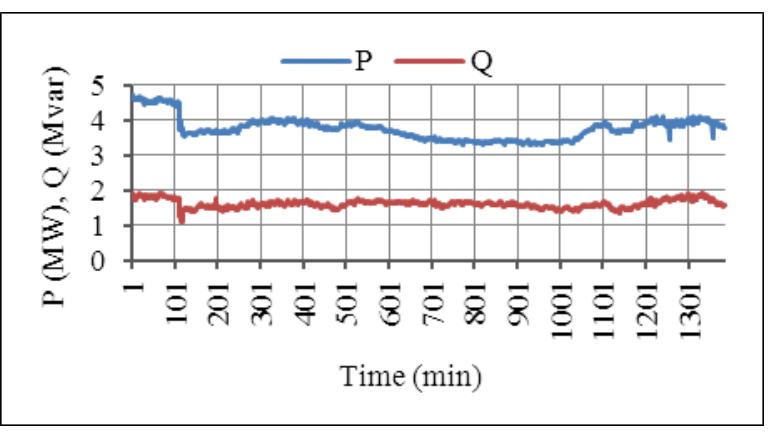

b)

Fig. 2. Variation of $\mathrm{P}$ and $\mathrm{Q}$ at node $\mathrm{Z}$

\section{The Harmonic Currents of Complex Load}

Below presented are the results of the analysis of currents of the complex load connected to the medium- and lowvoltage transformer windings. The shapes of curves of the measured currents are badly distorted. Over time, the shapes of the curves change but currents remain asymmetrical and nonsinusoidal. The measured harmonic currents represent series of values changing with time. Figure 3 shows a time series of effective value of the current and currents of the 3rd, 13th and 25th harmonics of the complex load at phase $\mathrm{C}$ of node $\mathrm{Z}$.

The analysis of the measured time series of harmonic currents shows that they are non-stationary. We present the results of the stationarity analysis of the time series for the effective value of the current (I) and 3rd, 13th and 25th harmonic currents at phase $\mathrm{C}$ for substation $\mathrm{Z}$ as an example.

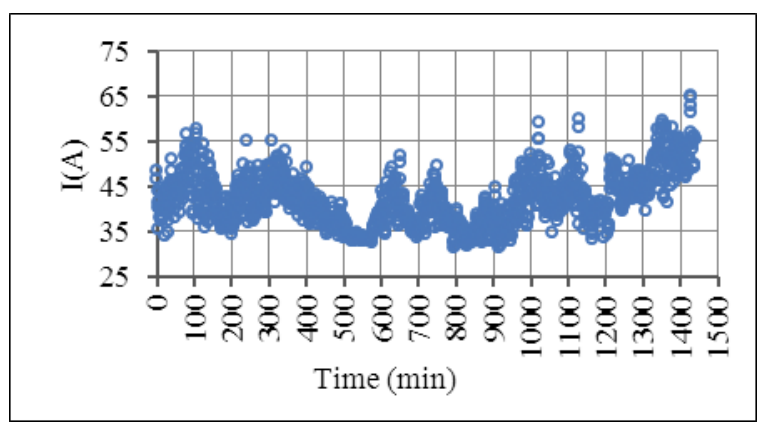

a)

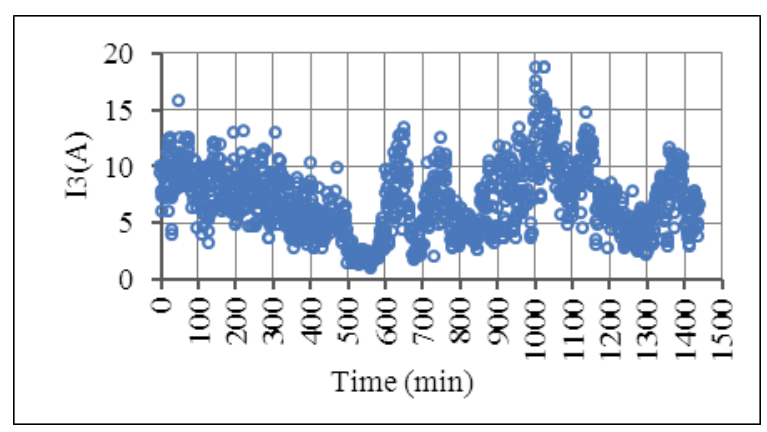

b)

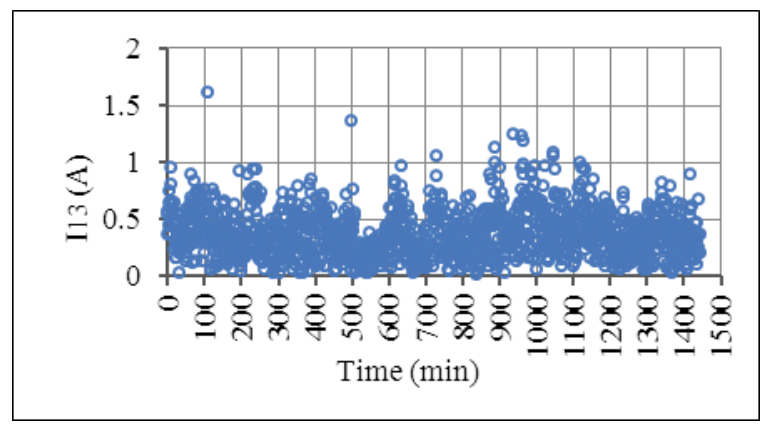

c)

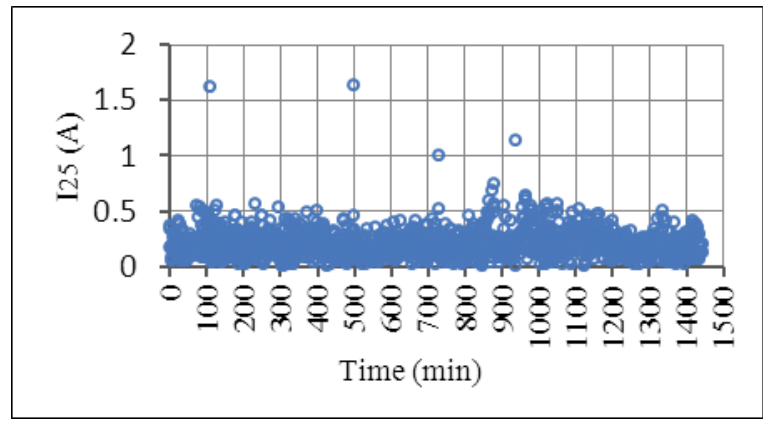

d)

Fig. 3. Scatter diagrams of currents of the load connected to both transformer windings

The time series were divided into 5 equal intervals of 288 elements each. The mean value and standard deviation were calculated for each interval and are presented in Table III, where mv - mean value, sd standard deviation. The data of the Table show that the mean values and standard deviation for each interval differ in value, which gives evidence of non-stationary time series.

With increase in the number of harmonic the time series become less variable which is seen from the comparison of scatter diagrams b, c, and d in Fig. 3. This conclusion is confirmed by the comparison of the mean values and standard deviation for the 3rd, 13th and 25th harmonics in Table III. Also, as the number of harmonic increases the mean values and standard deviation on the intervals differ by a smaller value. 
Table III. - Mean Values and Standard Deviations (A)

\begin{tabular}{|c|c|c|c|c|c|c|c|}
\hline \multicolumn{3}{|c|}{ Series interval } & 1 & 2 & 3 & 4 & 5 \\
\hline \multirow{2}{*}{\multicolumn{2}{|c|}{ I }} & $\mathrm{mv}$ & 43.23 & 39.70 & 38.35 & 41.68 & 46.60 \\
\hline & & $\mathrm{sd}$ & 4.73 & 5.03 & 3.87 & 5.30 & 5.94 \\
\hline \multirow{6}{*}{ 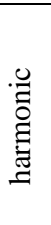 } & \multirow[b]{2}{*}{3} & $\mathrm{mv}$ & 8.45 & 4.79 & 5.77 & 9.09 & 6.16 \\
\hline & & sd & 1.97 & 2.32 & 2.62 & 3.19 & 2.17 \\
\hline & \multirow[t]{2}{*}{13} & $\mathrm{mv}$ & 0.39 & 0.31 & 0.33 & 0.45 & 0.34 \\
\hline & & sd & 0.21 & 0.19 & 0.19 & 0.23 & 0.17 \\
\hline & \multirow[t]{2}{*}{25} & $\mathrm{mv}$ & 0.22 & 0.19 & 0.2 & 0.25 & 0.19 \\
\hline & & sd & 0.14 & 0.14 & 0.11 & 0.15 & 0.09 \\
\hline
\end{tabular}

The mean value and standard deviation of the harmonic currents of a load of one phase are presented in Table IV. The mean values decrease with an increase in the harmonic number. They differ by more than 10 times for the 3rd and 25th harmonic currents.

Table IV. - Statistical Estimates of the Currents (A)

\begin{tabular}{|c|c|c|c|c|c|c|c|c|}
\hline \multirow{2}{*}{\begin{tabular}{c}
\multirow{2}{*}{ 节 } \\
\cline { 2 - 10 }
\end{tabular}} & \multicolumn{2}{|c|}{$\mathrm{M}$} & \multicolumn{2}{|c|}{$\mathrm{N}$} & \multicolumn{2}{c|}{$\mathrm{T}$} & \multicolumn{2}{c|}{$\mathrm{Z}$} \\
\cline { 2 - 10 } & $\mathrm{mv}$ & $\mathrm{sd}$ & $\mathrm{mv}$ & $\mathrm{ad}$ & $\mathrm{mv}$ & $\mathrm{sd}$ & $\mathrm{mv}$ & $\mathrm{sd}$ \\
\hline $\mathrm{I}$ & 45 & 17 & 35 & 12 & 293 & 162 & 38 & 10 \\
\hline 3 & 8.3 & 4.3 & 5.2 & 3.5 & 9.6 & 4.2 & 4.9 & 2.9 \\
\hline 5 & 7.2 & 2.2 & 4.5 & 1.3 & 9.7 & 2.0 & 4.6 & 1.5 \\
\hline 7 & 4.2 & 1.4 & 1.8 & 1.0 & 4.6 & 1.3 & 2.0 & 0.9 \\
\hline 9 & 2.0 & 0.9 & 1.0 & 0.5 & 2.5 & 1.1 & 1.0 & 0.6 \\
\hline 11 & 1.8 & 0.7 & 0.8 & 0.4 & 2.5 & 1.0 & 0.9 & 0.4 \\
\hline 13 & 1.2 & 0.5 & 0.5 & 0.3 & 1.7 & 0.7 & 0.7 & 0.3 \\
\hline 17 & 0.9 & 0.4 & 0.4 & 0.2 & 1.8 & 0.9 & 0.4 & 0.2 \\
\hline 19 & 0.9 & 0.4 & 0.4 & 0.2 & 1.8 & 1.0 & 0.4 & 0.2 \\
\hline 23 & 0.9 & 0.5 & 0.3 & 0.2 & 0.8 & 0.6 & 0.4 & 0.2 \\
\hline 25 & 0.6 & 0.3 & 0.3 & 0.2 & 0.5 & 0.3 & 0.4 & 0.2 \\
\hline
\end{tabular}

The value of the 3rd harmonic current makes up 25-30 percent of the fundamental harmonic current. In some cases, the graphs of the 3rd harmonic current (Fig. 3b) remind the graph of active power (Fig. 2a) or effective value of the current of the loads (Fig. 3a). This fact is confirmed by the values of correlation coefficients $\left(r_{P, I n}\right)$ between active power and harmonic current. The calculated coefficients of correlation are presented in Table V. The coefficient of correlation between active power and the 3rd harmonic current of load at node $\mathrm{Z}$ equal to 0.59 shows a strong relationship according to the Chaddock scale. There are only two coefficients in the Table that correspond to the moderate relationship between powers and harmonic currents on the Chaddock scale $\quad\left(0.3<r_{P, I n}<0.49\right)$. In most of the studied cases the coefficients of correlation between powers and harmonic currents are lower than 0.3 , which is indicative of a weak relationship and probabilistic character of the harmonic currents.

Scatter diagrams of the values of currents of the load connected to the low-voltage winding at node $\mathrm{Z}$ are presented in Fig. 4. Change in the effective value of the current is smooth unlike that of the effective value of the current in Fig. 3a. The values of currents decreased by 2 times. The 3rd harmonic current is sharply variable (Fig. $4 b)$ but starting with the 13th harmonic the change in the currents becomes smooth (Fig. 4c).
Table V. - Correlation Coefficients between $P$ and $I n$

\begin{tabular}{|c|c|c|c|c|}
\hline \multirow{2}{*}{$\begin{array}{c}\text { har- } \\
\text { mo- } \\
\text { nic }\end{array}$} & $\mathrm{M}$ & $\mathrm{N}$ & $\mathrm{T}$ & $\mathrm{Z}$ \\
\cline { 2 - 5 } & -0.22 & $\mathbf{0 . 7 1}$ & -0.07 & $\mathbf{0 . 5 9}$ \\
\hline 3 & 0.01 & 0.26 & $\mathbf{0 . 4 3}$ & 0.26 \\
\hline 5 & 0.22 & $\mathbf{0 . 6 4}$ & 0.23 & $\mathbf{0 . 6 2}$ \\
\hline 7 & 0.12 & $\mathbf{0 . 3 6}$ & 0.25 & 0.29 \\
\hline 9 & -0.08 & -0.08 & 0.06 & 0.09 \\
\hline 11 & 0.02 & 0.11 & -0.09 & 0.29 \\
\hline 13 & -0.24 & 0.12 & -0.06 & 0.13 \\
\hline 17 & 0.02 & 0.08 & -0.19 & -0.05 \\
\hline 19 & -0.16 & -0.07 & -0.31 & -0.07 \\
\hline 23 & -0.33 & 0.01 & -0.20 & -0.12 \\
\hline 25 & & & & \\
\hline
\end{tabular}

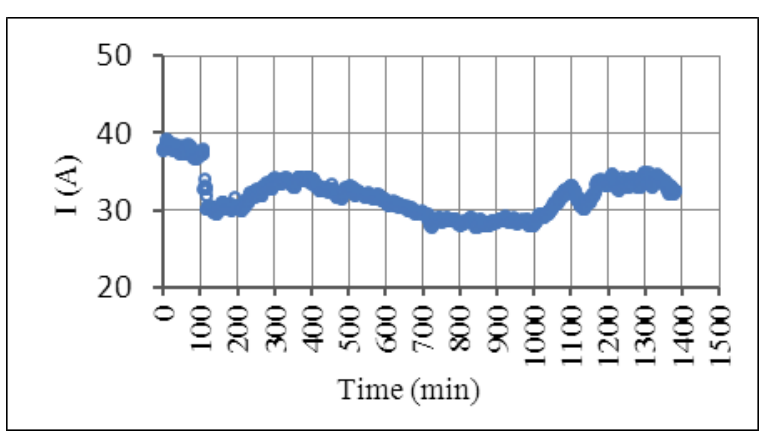

a)

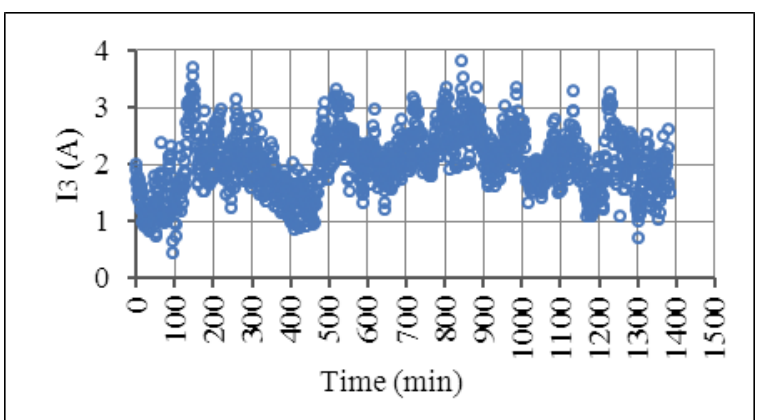

b)

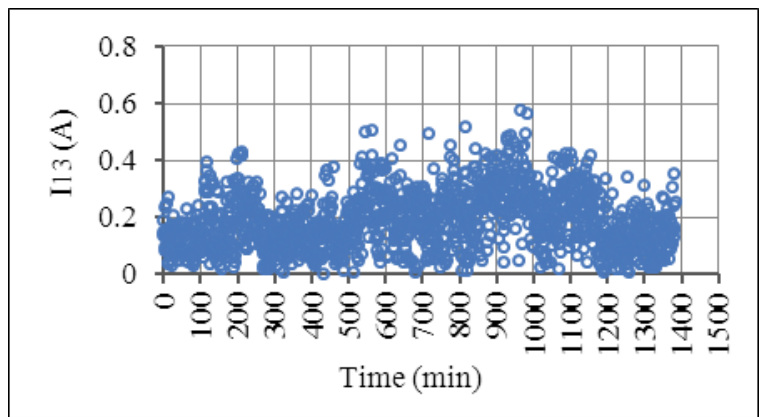

c)

Fig. 4. Scatter diagrams of currents of the load connected to the low-voltage winding

\section{Analysis of the Interrelation between Voltages and Currents of the Harmonics}

The analysis is made for the harmonic currents of a complex load connected to the medium- and low- voltage 
transformer windings. The values of harmonic voltages are determined by the values of harmonic currents of loads and transmission lines that are connected to the node. In order to find out the influence of harmonic currents of loads on the values of respective voltages at the studied nodes of the network, we calculated the coefficients of correlation between voltage and current of a corresponding load harmonic for three phases. Table VI presents the coefficients for one phase, in which their values are the highest. The values of the correlation coefficients, which correspond to the moderate and strong relationships on the Chaddock scale, are highlighted in bold. The Table shows that only three coefficients have the values that correspond to the moderate and strong relationship, i.e. $r_{U n, I n}>0.5$. Seven coefficients have the values demonstrating a moderate relationship $\left(0.3<r_{U n, I n}<0.49\right)$. The overwhelming majority of coefficients testify to a weak relationship between voltages and currents of the similar harmonics. The obtained result is indicative of the fact that the formation of harmonic voltages at nodes is greatly affected not only by the currents of loads, but also by the currents of transmission lines from neighboring substations.

Table VI. - Correlation Coefficients between $U n$ and In

\begin{tabular}{|c|c|c|c|c|}
\hline \multirow{2}{*}{$\begin{array}{c}\text { har- } \\
\text { mo- } \\
\text { nic }\end{array}$} & $\mathrm{M}$ & $\mathrm{N}$ & $\mathrm{T}$ & $\mathrm{Z}$ \\
\hline 3 & 0.13 & 0.02 & $\mathbf{0 . 6 3}$ & -0.11 \\
\hline 5 & 0.41 & 0.42 & 0.45 & 0.03 \\
\hline 7 & 0.03 & -0.14 & 0.35 & 0.03 \\
\hline 9 & -0.06 & -0.08 & 0.39 & 0.01 \\
\hline 11 & -0.04 & -0.09 & 0.29 & 0.13 \\
\hline 13 & 0.16 & 0.01 & -0.03 & 0.16 \\
\hline 17 & 0.00 & 0.00 & 0.19 & 0.29 \\
\hline 19 & 0.12 & 0.02 & 0.42 & 0.30 \\
\hline 23 & 0.15 & -0.01 & 0.24 & $\mathbf{0 . 5 3}$ \\
\hline 25 & 0.38 & 0.08 & 0.14 & $\mathbf{0 . 5 1}$ \\
\hline
\end{tabular}

\section{E. Histograms of the Harmonic Currents}

The graphs of powers and currents as well as the studied correlation relationships demonstrate the probabilistic character of harmonic conditions. We used histograms to get an idea about the distribution function of measured harmonic currents. The histograms are constructed for the current of the complex load connected to both transformer windings. Figure 5 presents the histograms of the 5 th and 17 th harmonic complex load currents of phase A at node $\mathrm{N}$.

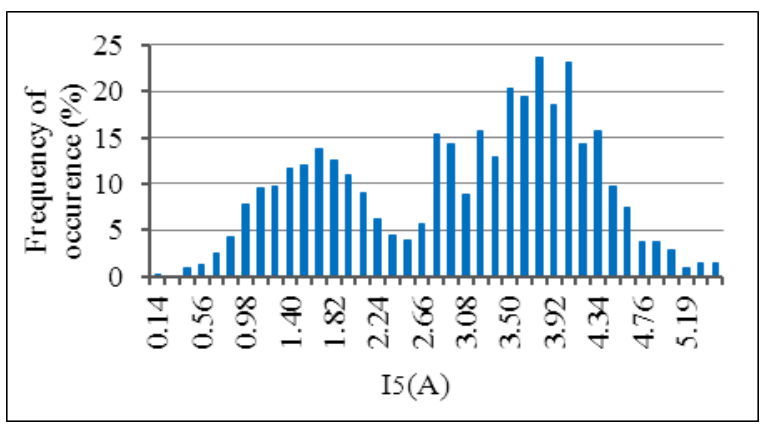

a)

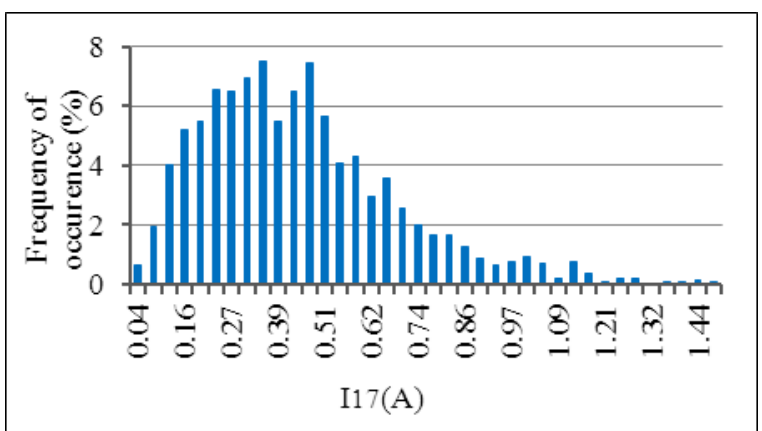

b)

Fig. 5. Histograms of the harmonic currents

The histogram of the 5th harmonic current has two peaks and consists of two histograms that are close in form to the Gaussian distribution. The histogram of the 17th harmonic is close to the Rayleigh distribution. The choice of the appropriate models describing the distributions of probabilities of measured harmonic currents is to be made further in the analysis.

\section{F. Phase Angles of the Currents}

Phase angles of load currents were investigated with the aid of scatter diagrams on a complex plane. Figure 6 presents scatter diagrams of the effective value of current of the load connected to both transformer windings, at phase $\mathrm{C}$ of node $\mathrm{Z}$ and its components of the $3 \mathrm{rd}$ and 13th harmonic, where a - active current, $\mathrm{r}$ - reactive current. Before the 13th harmonic the scatter diagrams of phase angles of currents have an oblong form and respective distribution as in Figs. $6 \mathrm{a}$ and $6 \mathrm{~b}$. For the 13th and higher harmonics the distributions of phase angles take the shape close to a circle with respect to the beginning of the complex plane coordinates as in Fig. 6c for the 13th harmonic.

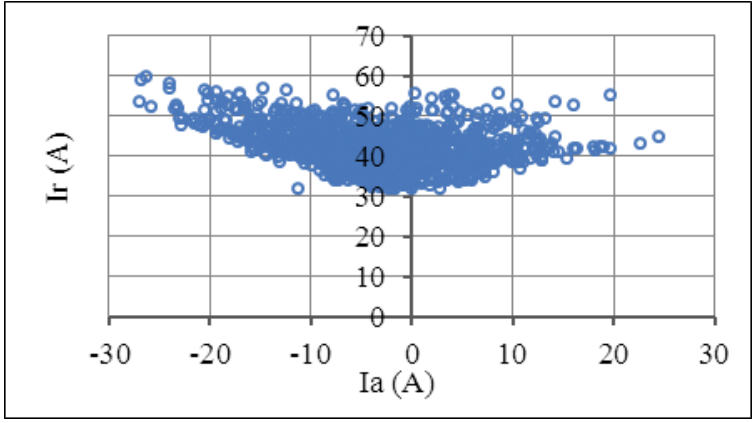

a)

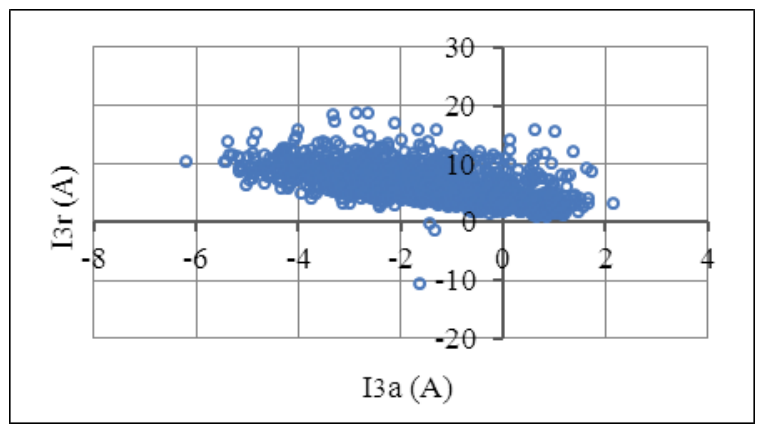

b) 


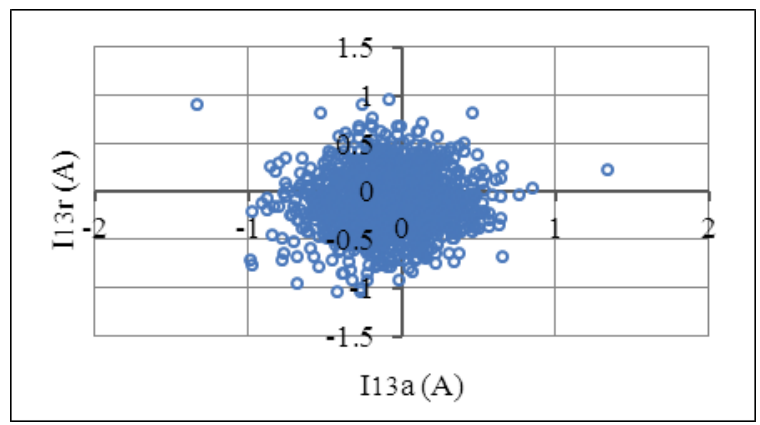

c)

Fig. 6. Scatter diagrams of phase angles of currents of the load connected to both transformer windings

Figure 7 presents phase angles of the currents of the load connected to the low-voltage transformer winding at node $Z$. Phase angles of the effective current of the load (Fig. 7a) lie in the range $\pi / 2<\theta_{h}<\pi$. Phase angles of the 3rd harmonic current (Fig. 7b) have distribution close to the shape of a circle but shifted with respect to the beginning of the coordinates of the complex plane. Starting with the 13th harmonic the distribution of phase angles has the shape of a circle located symmetrically with respect to the beginning of the coordinates of the complex plane. The phase angles lie in the range $0<\theta_{h}<2 \pi$.

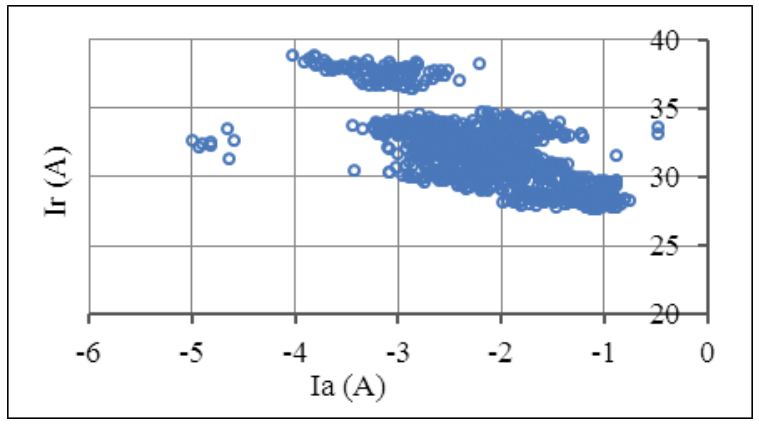

a)

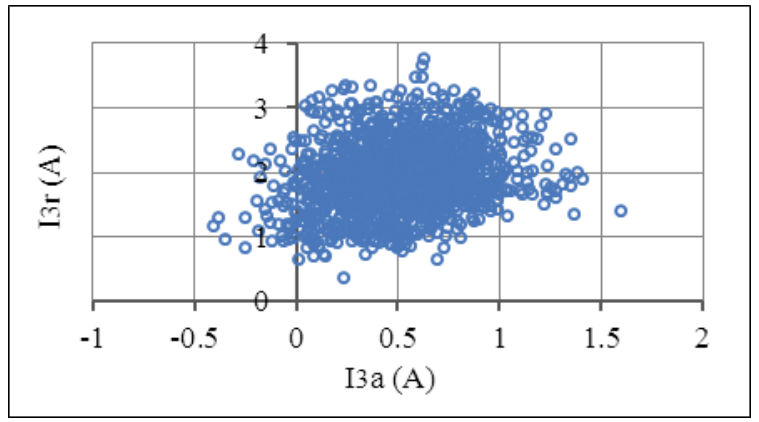

b)

Fig. 7. Scatter diagrams of phase angles of the currents of the load connected to the low-voltage transformer winding

\section{Conclusion}

At all the studied nodes the limits of harmonic voltages are exceeded. The voltages are normally exceeded at harmonics $3,5,7,9,23$.
Harmonic load currents are of a probabilistic character. They are weakly correlated with the powers of loads and harmonic voltages at nodes. The values of harmonic currents decrease as the harmonic numbers increase.

The probability distributions of harmonic currents have one- and two- peak histograms. Their forms are close to the Gaussian distribution and to the distribution of Rayleigh but require the specifying studies.

Phase angles of harmonic currents normally lie in the range $0<\theta_{h}<2 \pi$.

\section{Acknowledgement}

The work was supported by the grant of the President of the Russian Federation for the Leading Scientific School No. 4711.2014.8.

\section{References}

[1] T.C. Shuter, H. T. Vollkommer, T.L. Kirkpatrick, "A survey of harmonic levels on the American electric power distribution system ", IEEE Trans. on Power Delivery, Vol. 4, No. 4, October 1989, pp. 2204-2213.

[2] A.E. Emanuel, J.A.Orr, D.Cyganski, E.M.Gulachenski, “A survey of harmonic voltages and currents at distribution substations", IEEE Trans. on Power Delivery, Vol. 6, No. 4, October 1991, pp. 1883-1890.

[3] A.E. Emanuel, J.A.Orr, D.Cyganski, E.M.Gulachenski, “A survey of harmonic voltages and currents at the customer's bus", IEEE Trans. on Power Delivery, Vol. 8, No. 1, January 1993, pp. 411-421.

[4] Chung-Hsing Hu, Chi-Jui Wu, Shih-Shong Yen, Yu-Wu Chen, Bor-An Wu, Jan-San Hwang, "Survey of harmonic voltage and current at distribution substation in Northern Taiwan", IEEE Trans. on Power Delivery, Vol. 12, No. 3, July 1997, pp. 1275-1284.

[5] I. M. Nejdawi, A.E. Emanuel, D.J.Pileggi, M.J. Corridori, R.D. Archambeault, "Harmonics trend in USA: a preliminary survey”, IEEE Trans. on Power Delivery, Vol. 14, No. 4, Oktober 1999, pp. 1488-1494.

[6] A.B. Vasconcellos, T. I. R. de C. Malheiro, B.C. Castillo, A. T. da Silva, A. V. Festa, F.L. Gomes, "Analysis of power quality in a system of relay TV", in Proc. 14th ICHQP, Bergamo, Italy, 26-29 September, 2010.

[7] I.S.Fardiev, Y.A.Vasiliev, V.M.Meyer, "Energy saving lamps and their impact on power quality of the supply network", Energetika Tatarstana. 2009, No.4, pp. 8-12.

[8] R. Hartungi, L. Jiang, "Investigation of power quality in health care facility", in Proc. ICREPQ, Granada, Spain, 23-25 March, 2010

[9] V.S.Borovikov, M.V.Volkov, V.V.Ivanov, V.V.Litvak, V.A.Melnikov, A.I.Pogonin, N.N.Kharlov, "Experience of corporate examination of the $110 \mathrm{kV}$ electrical networks of Siberia", Tomsk, Publishing House of Tomsk Polytechnic University, 2010.

[10] State standard R 54149-2010. Electric energy. Electromagnetic compatibility of technical equipment. Power quality limits in the public power supply systems. Moskva. Standartinform. 2012.

[11] EN 50160: 2010. Voltage characteristics of electricity supplied by public electricity networks. 